\title{
A viscous blast-wave model for high energy heavy-ion collisions
}

\author{
Amaresh Jaiswal ${ }^{1, a}$ and Volker Koch ${ }^{2}$ \\ ${ }^{1}$ GSI, Helmholtzzentrum für Schwerionenforschung, Planckstrasse 1, D-64291 Darmstadt, Germany \\ ${ }^{2}$ Lawrence Berkeley National Laboratory, Nuclear Science Division, MS 70R0319, Berkeley, California \\ 94720, USA
}

\begin{abstract}
Employing a viscosity-based survival scale for initial geometrical perturbations formed in relativistic heavy-ion collisions, we model the radial flow velocity at freeze-out. Subsequently, we use the Cooper-Frye freeze-out prescription, with viscous corrections to the distribution function, to extract the transverse momentum dependence of particle yields and flow harmonics. We fit the model parameters for central collisions, by fitting the spectra of identified particles at the Large Hadron Collider (LHC), and estimate them for other centralities using simple hydrodynamic relations. We use the results of Monte Carlo Glauber model for initial eccentricities. We demonstrate that this improved viscous blast-wave model leads to good agreement with transverse momentum distribution of elliptic and triangular flow for all centralities and estimate the shear viscosity to entropy density ratio $\eta / s \simeq 0.24$ at the LHC.
\end{abstract}

\section{Introduction}

The quark-gluon plasma (QGP) formed in relativistic heavy-ion collisions exhibit strong collective behaviour and hence can be studied within the framework of relativistic hydrodynamics. The hydrodynamical modelling of heavy-ion collisions suggests that the QGP behaves like a nearly perfect fluid having an extremely small shear viscosity to entropy density ratio $\eta / s$ [1-3]. Apart from hydrodynamics, the collective behaviour of QGP can also be studied within the so-called blast-wave model. Schnedermann et. al. used the blast-wave model for the first time to fit the transverse momentum spectra using a simple functional form for the phase-space density distribution at kinetic freeze-out [4]. They approximated hydrodynamical results with only two parameters: a kinetic temperature, and a radial flow strength. Subsequently, there were several improvements incorporated in this model to account for the anisotropies in the transverse flow profile [5,6]. Within the blast-wave model, Teaney made the first attempt to estimate the effect of shear viscosity on spectra and elliptic flow [7]. However, the viscous effects were included only in the freeze-out prescription and the model parameters had to be fit separately for central and non-central collisions.

Here we generalize the blast-wave model to include viscous effects by using a viscosity-based survival scale for initial geometrical anisotropies, formed in relativistic heavy-ion collisions, to parametrize the radial flow velocity. Including $\eta / s$, the present model has five parameters which has to be fitted only for one centrality. We obtain obtain relations to account for the centrality dependence of model parameters, so that one only need to fit them for central collisions, rendering the model

ae-mail: jaiswal.amaresh@gmail.com 
with increased predictive power. We also consider the first-order viscous corrections to the distribution function in the Cooper-Frye freeze-out prescription for particle production, [7]. In essence, we provide a model which incorporates the key features of viscous hydrodynamic evolution without requiring to do the actual evolution. We employ this viscous blast-wave model to obtain the transverse momentum dependence of particle yields and flow harmonics for LHC. We fix the model parameters by fitting the transverse momentum distribution of identified particle spectra. Subsequently, we demonstrate that this leads to reasonably good agreement with transverse momentum dependence of elliptic and triangular flow for various centralities. Within the present model, we estimate the shear viscosity to entropy density ratio $\eta / s \simeq 0.24$ at the LHC.

\section{Blast wave model}

The blast-wave model has been used extensively to fit experimental data and it provides good description of spectra and elliptic flow observed in relativistic heavy-ion collisions [5-9]. The most important feature of the blast-wave model is the Hubble like parametrization of transverse velocity which is found to be in agreement with hydro results [10,11]. The model assumes boost invariance [12], as well as rotational invariance. We work in the Milne co-ordinate system where,

$$
\tau=\sqrt{t^{2}-z^{2}}, \quad \eta_{s}=\tanh ^{-1}(z / t), \quad r=\sqrt{x^{2}+y^{2}}, \quad \varphi=\operatorname{atan} 2(y, x) .
$$

Here the metric tensor for this co-ordinate system is $g_{\mu \nu}=\operatorname{diag}\left(1,-\tau^{2},-1,-r^{2}\right)$. The hydrodynamic fields are parametrized as [7]

$$
T=T_{f} \Theta(R-r), \quad u^{r}=u_{0} \frac{r}{R} \Theta(R-r), \quad u^{\varphi}=u^{\eta_{s}}=0, \quad u^{\tau}=\sqrt{1+\left(u^{r}\right)^{2}},
$$

where $R$ is the transverse radius of the fireball at freeze-out. The condition $u^{\mu} u_{\mu}=1$ leads to the expression for $u^{\tau}$. [13]

Using the Cooper-Frye prescription for particle production, the hadron spectra can be obtained as

$$
\frac{d N}{d^{2} p_{T} d y}=\frac{1}{(2 \pi)^{3}} \int p_{\mu} d \Sigma^{\mu} f(x, p),
$$

where $d \Sigma_{\mu}$ is the oriented freeze-out hyper-surface and $f(x, p)$ is the distribution function of the particles. The distribution function can be split as equilibrium and non-equilibrium parts, $f=f_{0}+\delta f$ where the equilibrium distribution function is given by

$$
f_{0}=\frac{1}{\exp \left(u_{\mu} p^{\mu} / T\right)+a},
$$

where $a=+1$ for baryons and $a=-1$ mesons. For a system close to equilibrium, i.e., $\delta f \ll f_{0}$, we employ the Grad's 14-moment approximation for the non-equilibrium part [14]

$$
\delta f=\frac{f_{0} \tilde{f}_{0}}{2(\epsilon+P) T^{2}} p^{\alpha} p^{\beta} \pi_{\alpha \beta} \quad \Rightarrow \quad \delta f_{1}=\frac{f_{0} \tilde{f}_{0}}{T^{3}}\left(\frac{\eta}{s}\right) p^{\alpha} p^{\beta} \nabla_{\langle\alpha} u_{\beta\rangle} .
$$

where $\tilde{f}_{0}=1-a f_{0}$ is the quantum factor, $\pi_{\alpha \beta}$ is the shear stress tensor, $\eta$ is the coefficient of shear viscosity, $s=(\epsilon+P) / T$ is the entropy density and the angular brackets denote traceless symmetric projection orthogonal to the fluid four-velocity [15]. In the second equality, we have approximated the shear stress tensor with its first-order relativistic Navier-Stokes expression, $\pi_{\alpha \beta}=2 \eta \nabla_{\langle\alpha} u_{\beta\rangle}$. In the case of blast-wave model, the form of $p^{\alpha} p^{\beta} \nabla_{\langle\alpha} u_{\beta\rangle}$ is calculated in Refs. [7, 16]. 
The anisotropic flow is defined as

$$
v_{n}\left(p_{T}\right) \equiv \frac{\int_{-\pi}^{\pi} d \phi \cos \left[n\left(\phi-\Psi_{n}\right)\right] \frac{d N}{d y p_{t} d p_{T} d \phi}}{\int_{-\pi}^{\pi} d \phi \frac{d N}{d y p_{t} d p_{T} d \phi}}
$$

where $\Psi_{n}$ is the event-plane angle for the $n$-th harmonic. Up to first order in viscosity [7],

$$
v_{n}\left(p_{T}\right)=v_{n}^{(0)}\left(p_{T}\right)\left(1-\frac{\int d \phi \frac{d N^{(1)}}{d y p_{t} d p_{T} d \phi}}{\int d \phi \frac{d N^{(0)}}{d y p_{t} d p_{T} d \phi}}\right)+\frac{\int d \phi \cos \left[n\left(\phi-\Psi_{n}\right)\right] \frac{d N^{(1)}}{d y p_{t} d p_{T} d \phi}}{\int d \phi \frac{d N^{(0)}}{d y p_{t} d p_{T} d \phi}}
$$

where the superscript '(0)' and '(1)' denote quantities calculated using the ideal distribution function, Eq. (4), and first-order viscous correction, Eq. (5), respectively.

\section{Viscous blast-wave model}

In terms of the Fourier expansion for a single-particle distribution, the definition of the participant anisotropies, $\varepsilon_{n}$, is

$$
f(\varphi)=\frac{1}{2 \pi}\left[1+2 \sum_{n=1}^{\infty} \varepsilon_{n} \cos \left[n\left(\varphi-\psi_{n}\right)\right]\right] .
$$

Here $\psi_{n}$ is the angle between the $x$ axis and the major axis of the participant distribution. The initial geometrical anisotropies, $\varepsilon_{n}$, eventually converts to anisotropies in the radial fluid velocity,

$$
u^{r}=u_{0} \frac{r}{R}\left[1+2 \sum_{n=1}^{\infty} u_{n} \cos \left[n\left(\varphi-\psi_{n}\right)\right]\right] .
$$

Next, we determine the conversion efficiency of the initial geometrical eccentricity to final anisotropy in the radial fluid velocity, $u_{n} / \varepsilon_{n}$.

In Ref. [17], a viscosity-based survival scale for anisotropic structures formed by point like perturbations was proposed using the dispersion relation for sound in a viscous medium,

$$
\omega=c_{s} k+i k^{2} \frac{1}{T}\left(\frac{2}{3} \frac{\eta}{s}\right)
$$

In the above equation, $c_{s}$ is the speed of sound in the medium and $\eta$ is the coefficients of shear viscosity. Using a Fourier ansatz, $\exp (i \omega t-i k x)$, we see that the amplitudes of the stress tensor harmonics, with momentum $k$, are attenuated by a factor

$$
\delta T^{\mu v}(t, k)=\exp \left[-\left(\frac{2}{3} \frac{\eta}{s}\right) \frac{k^{2} t}{T}\right] \delta T^{\mu v}(0, k)
$$

where we have ignored the oscillatory pre-factor. The presence of momentum squared in the exponent results in enhanced effect of viscosity for the higher harmonics. 
We note that each harmonics is essentially a damped oscillator with wave-vector $k$ whose amplitude is progressively suppressed due to viscous effects. They form standing waves on the fireball circumference throughout the evolution, i.e.,

$$
2 \pi R=n \frac{2 \pi}{k},
$$

where $R$ is the transverse radius of the fireball at freeze-out. Therefore, at the freeze-out time $t_{f}$, the wave amplitude reaction is

$$
\frac{\left.\delta T^{\mu v}\right|_{t=t_{f}}}{\left.\delta T^{\mu v}\right|_{t=0}}=\exp \left[-n^{2}\left(\frac{2}{3} \frac{\eta}{s}\right) \frac{t_{f}}{R^{2} T_{f}}\right],
$$

where $T_{f}$ is the freeze-out temperature.

The final radial flow velocity is due to initial geometrical perturbations and in absence of viscosity, the conversion efficiency remains the same for all harmonics. For a viscous medium, the conversion efficiency must be proportional to the wave amplitude reaction,

$$
\frac{u_{n}}{\varepsilon_{n}}=\alpha_{0} \exp \left[-n^{2}\left(\frac{2}{3} \frac{\eta}{s}\right) \frac{t_{f}}{R^{2} T_{f}}\right]
$$

where $\alpha_{0}$ is the constant of proportionality. In Ref. [18], Shuryak and Zahed proposed that the r.h.s. of Eq. (14) should be equal to $v_{n} / \varepsilon_{n}$ where $v_{n}$ is the final $p_{T}$-integrated flow harmonics. This assumption misses several key features of hydrodynamic evolution [19-21]. We therefore emphasize that the acoustic damping should be applicable to the hydrodynamic variables $u_{n}$ rather then to the final state observables $v_{n}[16]$.

\section{Initial conditions and numerical results}

In order to reduce the number of free parameters of the blast-wave model, we set-up initial conditions of the collisions. We consider two identical nuclei with mass number $A$ colliding at relativistic energies with impact parameter $b$. The radius $r_{0}$ of the circle, drawn in the transverse plane, which divides the boundary of the overlap zone equally in four parts is given by [16]

$$
r_{0}=\frac{1}{2}\left(b^{2}-2 b R_{0} \sqrt{2+\frac{b}{R_{0}}}+4 R_{0}^{2}\right)^{1 / 2},
$$

where $R_{0}=1.25 A^{1 / 3} \mathrm{fm}$ is the radius of each colliding nuclei. This is done in order to approximate the boundary of the overlap zone as the second harmonics of initial geometrical fluctuations $\varepsilon_{2}$, on $r_{0}$. Note that $\varepsilon_{2}$ is the most prominent eccentricity for non-central collisions.

The transverse expansion of the fireball is obtained by using the radial velocity, Eq. (2),

$$
u^{r} \equiv \frac{d r}{d \tau}=u_{0} \frac{r}{R} \Rightarrow \int_{r_{0}}^{R} \frac{d r}{r}=\int_{0}^{\tau_{f}} \frac{u_{0}}{R} d \tau \Rightarrow R=r_{0} \exp \left(\frac{u_{0} \tau_{f}}{R}\right)
$$

which can be solved for $R$ given the freeze-out time $\tau_{0}$ and the isotropic expansion velocity $u_{0}$. Next we determine the freeze-out times for non-central collisions when it is fixed for the central one. To this end, we employ the analytical result of Bjorken expansion $\epsilon \propto \tau^{-4 / 3}$. Assuming the initial thermalization time and the freeze-out energy density to be same for all collisions, we get

$$
\tau_{f}=\tau_{f 0}\left(\frac{\epsilon_{i}}{\epsilon_{i 0}}\right)^{3 / 4}
$$



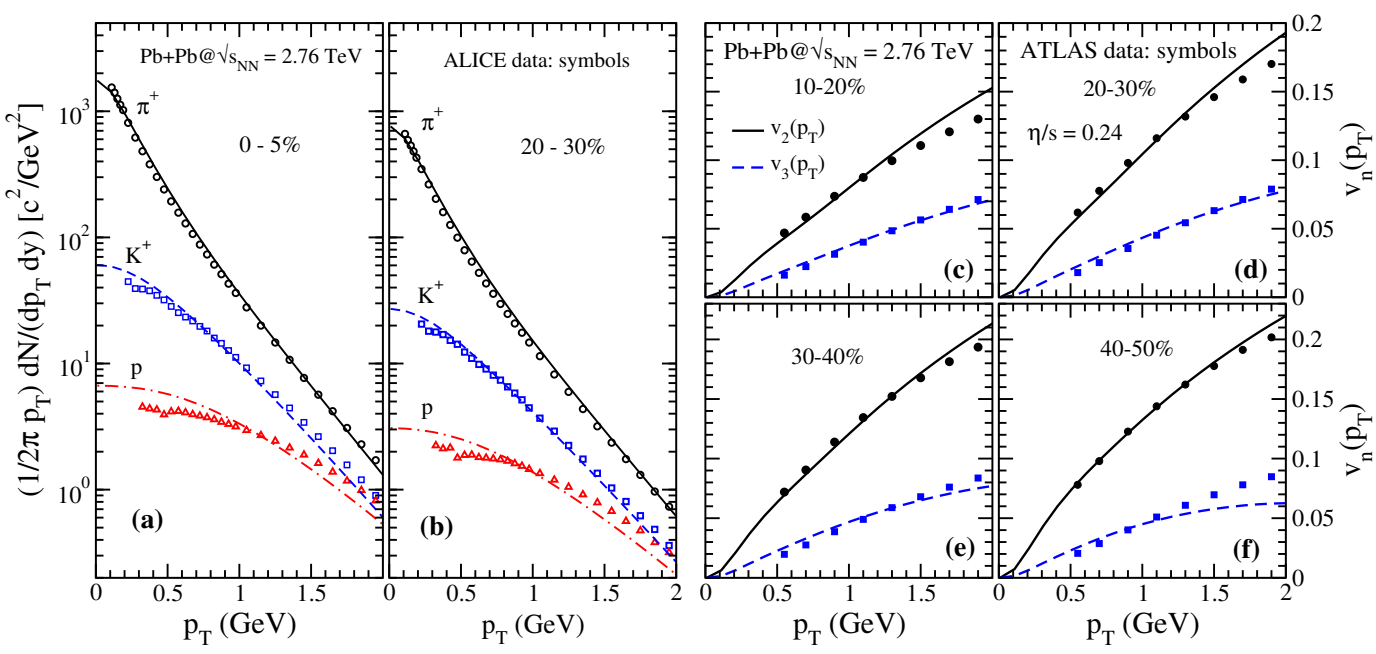

Figure 1. Transverse momentum distribution of particle multiplicities in $\mathrm{Pb}+\mathrm{Pb}$ collisions at $\sqrt{s_{N N}}=2.76 \mathrm{TeV}$. We show results for $\pi^{+}, K^{+}$, and $p$ in two centrality ranges, (a): $0-5 \%$ and (b): $20-30 \%$. The symbols represent ALICE data [22] at midrapidity and the lines correspond to viscous blast-wave calculations. (c)-(f): Transverse momentum dependence of the anisotropic flow coefficients $v_{n}\left(p_{T}\right)$ of charged hadrons, for $n=2$ and 3 , calculated at various centralities in $\mathrm{Pb}+\mathrm{Pb}$ collisions at $\sqrt{s_{N N}}=2.76 \mathrm{TeV}$ in the viscous blast-wave model (lines) with $\eta / s=0.24$ as compared to the ATLAS data [23] (symbols).

Here $\tau_{f 0}$ is the freeze-out time for most central collisions, which is fixed by fitting the corresponding transverse momentum spectra. The ratio $\epsilon_{i} / \epsilon_{i 0}$ is the initial central energy density for all centrality, scaled by its corresponding value in most central collisions, and can be determined using an initial condition model. Therefore the parameters that needs to be fixed within the viscous-blast wave model, in order to fit the spectra, are the freeze-out temperature $T_{f}$, the freeze-out time for central collision $\tau_{f 0}$ and the maximum radial flow velocity $u_{0}$. An interplay between $\alpha_{0}$ in Eq. (14) and $\eta / s$ is important to reproduce the flow harmonics.

In Fig. 1, we show our results for transverse momentum distribution of identified particles and $v_{n}\left(p_{T}\right)$ for $\mathrm{Pb}+\mathrm{Pb}$ collisions at $\sqrt{s_{N N}}=2.76 \mathrm{TeV}$ and compare it with experimental data measured by the ALICE [22] and ATLAS [23] collaborations. For $\epsilon_{i} / \epsilon_{i 0}$ we use the results from the Glauber model for various centralities. Figure 1 shows the transverse momentum distribution of pions, kaons, and protons spectra for (a): $0-5 \%$ and (b): $20-30 \%$ centrality. We observe that, for a freeze-out temperature of $120 \mathrm{MeV}$, the spectra for $\pi^{+}$and $K^{+}$from the viscous blast-wave model are in good overall agreement with the ALICE data. On the other hand, the freeze-out temperature for protons is considered to be $135 \mathrm{MeV}$ in order to fit the experimental data. The freeze-out time $\tau_{f 0}=8 \mathrm{fm}$ was used to fit $0-5 \%$ most central collision data. Figure 1 (c)-(f) shows our results for the $v_{n}\left(p_{T}\right)$, for various centralities, in comparison with the ATLAS data [23]. We find fairly good agreement with the experimental data for elliptic flow, $v_{2}\left(p_{T}\right)$, and triangular flow, $v_{3}\left(p_{T}\right)$, by choosing $\alpha_{0}=0.4$ and $\eta / s=0.24$. We have used the root-mean square values of the eccentricities obtained in the MC-Glauber model for initial eccentricity $\varepsilon_{n}$ [24].

\section{Conclusions}

We have generalized the blast-wave model to incorporate viscous effects by using a viscosity-based survival scale for initial geometrical anisotropies formed in relativistic heavy-ion collisions. This 
viscous damping formula is introduced in the parametrization of the radial flow velocity. Including $\eta / s$, the viscous blast wave model presented here involved five parameters which has to be fitted for only one centrality. This model therefore incorporates the key features of viscous hydrodynamic evolution without requiring to do the actual evolution. This model was used to obtain the transverse momentum distribution of particle yields and anisotropic flow at LHC. The model parameters were fixed by fitting the transverse momentum spectra of identified particles. We showed that, for various centralities, a fairly good agreement was achieved for transverse momentum distribution of elliptic and triangular flow. Within the present model, we estimated the ratio of shear viscosity to entropy density $\eta / s \simeq 0.24$ at the LHC.

Acknowledgements:- A.J. was supported by the Frankfurt Institute for Advanced Studies (FIAS), Germany. The work of V.K. was supported by the ExtreMe Matter Institute (EMMI) and by the Office of Nuclear Physics in the US Department of Energy's Office of Science under Contract No. DE-AC02-05CH11231.

\section{References}

[1] P. Romatschke and U. Romatschke, Phys. Rev. Lett. 99, 172301 (2007).

[2] H. Song and U. W. Heinz, Phys. Rev. C 77, 064901 (2008).

[3] R. S. Bhalerao, A. Jaiswal and S. Pal, Phys. Rev. C 92, 014903 (2015).

[4] E. Schnedermann, J. Sollfrank and U. W. Heinz, Phys. Rev. C 48, 2462 (1993).

[5] P. Huovinen, P. F. Kolb, U. W. Heinz, P. V. Ruuskanen and S. A. Voloshin, Phys. Lett. B 503, 58 (2001).

[6] C. Adler et al. [STAR Collaboration], Phys. Rev. Lett. 87, 182301 (2001).

[7] D. Teaney, Phys. Rev. C 68, 034913 (2003).

[8] Z. Tang, Y. Xu, L. Ruan, G. van Buren, F. Wang and Z. Xu, Phys. Rev. C 79, 051901 (2009).

[9] X. Sun, H. Masui, A. M. Poskanzer and A. Schmah, Phys. Rev. C 91, 024903 (2015).

[10] D. Teaney, J. Lauret and E. V. Shuryak, nucl-th/0110037.

[11] M. Habich, J. L. Nagle and P. Romatschke, Eur. Phys. J. C 75, 15 (2015).

[12] J. D. Bjorken, Phys. Rev. D 27, 140 (1983).

[13] F. Cooper and G. Frye, Phys. Rev. D 10, 186 (1974).

[14] H. Grad, Comm. Pure Appl. Math. 2, 331 (1949).

[15] R. S. Bhalerao, A. Jaiswal, S. Pal and V. Sreekanth, Phys. Rev. C 89, 054903 (2014).

[16] A. Jaiswal and V. Koch, arXiv:1508.05878 [nucl-th].

[17] P. Staig and E. Shuryak, Phys. Rev. C 84, 034908 (2011).

[18] E. Shuryak and I. Zahed, Phys. Rev. C 88, 044915 (2013).

[19] R. A. Lacey, Y. Gu, X. Gong, D. Reynolds, N. N. Ajitanand, J. M. Alexander, A. Mwai and A. Taranenko, arXiv:1301.0165.

[20] R. A. Lacey, A. Taranenko, J. Jia, D. Reynolds, N. N. Ajitanand, J. M. Alexander, Y. Gu and A. Mwai, Phys. Rev. Lett. 112, 082302 (2014).

[21] R. A. Lacey, D. Reynolds, A. Taranenko, N. N. Ajitanand, J. M. Alexander, F. H. Liu, Y. Gu and A. Mwai, arXiv:1311.1728 [nucl-ex].

[22] B. Abelev et al. [ALICE Collaboration], Phys. Rev. C 88, 044910 (2013).

[23] G. Aad et al. [ATLAS Collaboration], Phys. Rev. C 86, 014907 (2012).

[24] E. Retinskaya, M. Luzum and J. Y. Ollitrault, Phys. Rev. C 89, 014902 (2014). 